Eur J Clin Chem Clin Biochem

1995; 33:433-439

(c) 1995 Walter de Gruyter \& Co.

Berlin - New York

\title{
Clinical Utility of a Wheat-Germ Precipitation Assay for Determination of Bone Alkaline Phosphatase Concentrations in Patients with Different Metabolic Bone Diseases
}

\author{
By Vania Braga ${ }^{1}$, Romolo Dorizzi ${ }^{2}$, Giorgio Brocco ${ }^{3}$, Maurizio Rossini $^{1}$, Nicoletta Zamberlan ${ }^{1}$, Davide Gatti ${ }^{1}$ and \\ Silvano Adami ${ }^{1}$ \\ 1 Cattedra di Reumatologia. Università di Verona, Verona, Italy \\ 2 Laboratorio analisi, Ospedale di Legnago, Legnago, Verona, Italy \\ 3 Istituto di Chimica e Microscopia Clinica, Policlinico, Verona, Italy
}

(Received January 31/April 24, 1995)

Summary: Bone alkaline phosphatase was evaluated by wheat-germ lectin precipitation in several clinical conditions. The study included 33 premenopausal healthy women, 46 postmenopausal apparently healthy women, 19 growing children, 24 patients with Paget's disease, 31 patients with primary hyperparathyroidism and 66 patients with hepatobiliary diseases.

In postmenopausal women the mean $\mathrm{T}$ score (i. e.: the number of SD below or above the mean for premenopausal women) was $2.6 \pm 1.3(\mathrm{SD})$ for bone alkaline phosphatase and $1.61 \pm 1.21$ for total alkaline phosphatase $(\mathrm{p}<0.001)$. The $\mathrm{T}$ score for bone alkaline phosphatase provided a better discrimination from normals for both Paget's disease $(22.1 \pm 27.8$ versus $12.8 \pm 16 \mathrm{p}<0.001)$ and primary hyperparathyroidism $(8.2 \pm 4.3$ versus $4.6 \pm 3.7 \mathrm{p}<0.005$ for bone alkaline phosphatase and total alkaline phosphatase respectively).

After treatment with intravenous bisphosphonate the percent decrease of bone alkaline phosphatase was larger than that of total alkaline phosphatase both in patients with Paget's disease $(-46 \%$ versus $-72 \% p<0.01)$ and in patients with primary hyperparathyroidism $(-21 \%$ versus $-47 \% \mathrm{p}<0.02)$ and an estimate of the precision $(\Delta$ mean/SD of the $\Delta$ mean) for bone alkaline phosphatase was 1.9-3.7 times higher than that of total alkaline phosphatase. In twelve osteoporotic patients treated for six months with oral alendronate the decrease in bone turnover was detected with significantly higher precision with bone alkaline phosphatase than with total alkaline phosphatase $(p<0.001)$.

In serum samples from patients with severe metabolic bone diseases without relevant hepatobiliary involvement, it was found that lectin was not able to fully precipitate bone alkaline phosphatase when its concentration was higher than $1000 \mathrm{U} / 1$. Serum dilution is therefore recommended before alkaline phosphatase separation.

\section{Introduction}

Total alkaline phosphatase ${ }^{1}$ ) activity is a valuable clinical marker for diagnosis and monitoring of severe bone disorders such as Paget's disease, where elevated alka-

\footnotetext{
') Enzyme:

Alkaline phosphatase (Orthophosphoric-monoester phosphohydrolase (alkaline optimum); EC 3.1.3.1)
}

line phosphatase values are due to increased activity of the skeletal or bone isoform. However, its use in monitoring the effect of treatment of less severe bone metabolic disorders such as osteoporosis is rather limited, since the enzyme activity of bone origin represents only a part of serum total alkaline phosphatase.

Recent studies indicate that the quantification of skeletal alkaline phosphatase activity in serum may provide a 
better marker for the rate of bone formation than total alkaline phosphatase $(1,2)$. The isoforms from liver, bone and kidney are derived from the same gene (3) and differ only on their tertiary structure as a consequence of postranslational modification, such as glycosylation and sialylation (3). It has been found to be difficult to distinguish and quantitate the skeletal isoform activity from alkaline phosphatase from other organs and tissues. The methods used in the past were either imprecise (heat-inactivation) (4) or cumbersome (electrophoresis) (5). More recently it has been found that the lectin wheat-germ agglutinin preferentially precipitates serum bone alkaline phosphatase (6). Both the total alkaline phosphatase and the non-precipitated alkaline phosphatase can be measured by conventional colorimetric methods, and bone alkaline phosphatase calculated from these two determinations.

We have used this wheat germ agglutinin assay to measure bone and non-bone alkaline phosphatase in normal subjects and in patients with liver or bone diseases. The effect of menopause and bisphosphonate therapy on alkaline phosphatase isoenzymes were also evaluated in order to assess the ability of the wheat-germ lectin precipitation to detect the changes in bone alkaline phosphatase occurring within the normal range for total alkaline phosphatase.

\section{Materials and Methods}

\section{Measurement of bone alkaline phosphatase}

Bone alkaline phosphatase was measured using the commercial kit "Test-combination of isoenzymes of alkaline phosphatase" (Boehringer Mannheim GmbH Diagnostica, Germany; lot N. 64943101) which includes wheat-germ lectin $(\geq 2 \mathrm{~g} / \mathrm{l})$ for precipitation of bone alkaline phosphatase dissolved in $5 \mathrm{mmol} / \mathrm{l}$ acetate buffer $\mathrm{pH}$ 4.5 , containing Triton X-100, $20 \mathrm{~g} / \mathrm{l}$, to prevent coprecipitation of biliary alkaline phosphatase.

The assay is performed as follows: after measuring the total alkaline phosphatase activity of the sample, $100 \mu \mathrm{l}$ of precipitating lectin reagent are mixed with $100 \mu \mathrm{l}$ of serum, incubated for 30 minutes at room temperature and centrifuged at $10000 \mathrm{~g}$ for 2.5 minutes. The residual alkaline phosphatase activity is then measured in the supernatant after multiplying this by two (for correction of the dilution factor) (7).

On the basis of studies comparing lectin precipitation with electrophoretic procedures for enzyme separation and of dilution factors, the following correcting function is suggested (7):

bone alkaline phosphatase $(U / 1)=1.118 \times$ total alkaline phosphatase -2.35 activity in supernatant

\section{Measurement of total alkaline phosphatase}

Alkaline phosphatase activity was determined at $37^{\circ} \mathrm{C}$ according to the optimised DGKCH (Deutsche Gesellschaft für Klinische Chemie) method /U/l) (8). The two determinations (total and supernatant alkaline phosphatase) were performed on the BM/Hitachi System 704 analyzer (Boehringer Mannheim Automated Analysis).
Routine laboratory tests were performed by autoanalyzer methods (Hitachi-DAX). Serum calcium was adjusted to an albumin concentration of $42 \mathrm{~g} / \mathrm{l}(9)$. Intact serum parathyrin was measured by an immunoradiometric method (Allegro PTH, Nichols, USA; lot N. 500641).

The alkaline phosphatase results were also analyzed in terms of $T$ score. This was calculated from the mean values found in premenopausal women. The following formula was used:

$$
\mathrm{T} \text { score }=\frac{\text { Individual value }- \text { mean premenopausals }}{\text { SD of premenopausals }}
$$

In a limited number of the patients ( 5 normal premenopausal women, 20 patients with hepatobiliary diseases and in 7 patients with Paget's disease) bone alkaline phosphatase was also measured by a commercial immunoradiometric assay (Ostase, Hybritech, USA).

Statistical analysis includes the simple linear correlation, Student $t$ test for paired observations and non-parametric methods ( $t$ test for location and comparison of two samples, Statgraphic, Statgraph, USA).

\section{Patients}

This study includes several groups of healthy subjects and patients (tab. 1): pre- and postmenopausal women, prepubertal subjects and patients with bone and hepatobiliary diseases. Serum samples were kept at $-20^{\circ} \mathrm{C}$ up to the time of assay which was carried out within 12 months. The most relevant routine biochemical data of the patients are listed in table 2 . The control women were apparently healthy and were not on any therapy known to influence calcium or bone metabolism. The time since menopause ranged from 1 to 25 years.

The diagnosis of primary hyperparathyroidism was based on elevated serum calcium and immunoreactive parathyroid hormone. In most patients with Paget's disease the diagnosis was based on obvious clinical grounds but in 4 of them serum total alkaline phosphatase was within the normal range and the diagnosis was based on previous evidence of raised alkaline phosphatase and on radiographic and scintigraphic findings. None of the patients with metabolic bone diseases showed biochemical evidence of hepatic or biliary diseases (normal serum $\gamma$-glutamyltransferase and aminotransferases) (tab. 2).

The patients with hepatobiliary diseases had both biochemical and clinical evidence of intra-hepatic or extra-hepatic biliary obstruction. Four out of 31 of the female patients were premenopausal.

The routine biochemical profile of children and healthy women was normal (data not shown).

A group of patients with primary hyperparathyroidism was also evaluated 2-4 weeks after a treatment course with alendronate. Twelve patients were given alendronate $5 \mathrm{mg}$ /day dissolved in 250 $\mathrm{ml}$ of saline solution for $5-7$ days and 7 patients received a single i. v. injection of $2.5 \mathrm{mg}$ alendronate.

Ten of the patients with Paget's disease were also studied 4 months after a treatment course with i. $v$. alendronate $(5 \mathrm{mg} /$ day for $2-4$ days). Twelve patients with lumbar spine bone mineral density more than $2 \mathrm{SD}$ below the reference range for young premenopausal women were studied before and 6 months after treatment with oral alendronate $20 \mathrm{mg} /$ day. The details pertaining this study have been published elsewhere (10).

\section{Results}

Three serum samples with low, normal and high bone alkaline phosphatase values were measured at five dif- 
Tab. 1 Main anagraphic data and mean ( \pm SEM) values of total alkaline phosphatase and its fractions in the different groups of patients (upper reference limit provided with the kit was $240 \mathrm{U} / 1$ for total alkaline phosphatase).

\begin{tabular}{|c|c|c|c|c|c|c|}
\hline & Children & $\begin{array}{l}\text { Pre- } \\
\text { menopausal } \\
\text { women }\end{array}$ & $\begin{array}{l}\text { Post- } \\
\text { menopausal } \\
\text { women }\end{array}$ & $\begin{array}{l}\text { Paget's } \\
\text { disease }\end{array}$ & $\begin{array}{l}\text { Primary } \\
\text { hyperpara- } \\
\text { thyroidism }\end{array}$ & $\begin{array}{l}\text { Hepato- } \\
\text { biliary } \\
\text { disease }\end{array}$ \\
\hline$N$ & 19 & 33 & 46 & 24 & 31 & 66 \\
\hline Male/female & $12 / 7$ & & & $15 / 9$ & $8 / 23$ & $35 / 31$ \\
\hline Age range (a) & $1-14$ & $21-45$ & $48-75$ & $47-80$ & $44-80$ & $20-84$ \\
\hline $\begin{array}{l}\text { Total alkaline phosphatase } \\
\text { (U/I) }\end{array}$ & $742(137)$ & $93(4)$ & $129(4)$ & $391(76)$ & $195(15)$ & $565(53)$ \\
\hline $\begin{array}{l}\text { Supernatant alkaline } \\
\text { phosphatase (U/l) }\end{array}$ & $155 \quad(23)$ & $55(3)$ & $68(3)$ & $106(13)$ & 69 (5) & $476(49)$ \\
\hline $\begin{array}{l}\text { Bone alkaline phosphatase* } \\
\text { (U/l) }\end{array}$ & $647(138)$ & $32(4)$ & $66(2)$ & $307(74)$ & $137(15)$ & $71(8)$ \\
\hline
\end{tabular}

* calculated with the function provided by the commercial kit, for correcting the residual alkaline phosphatase (total alkaline phos-

phatase - supernatant alkaline phosphatase) to bone alkaline phosphatase.

Tab. 2 Laboratory values in bone and hepatobiliary diseases (mean \pm SD); serum calcium was corrected for albumin values.

\begin{tabular}{|c|c|c|c|c|c|}
\hline Analyses & $\begin{array}{l}\text { Reference } \\
\text { range }\end{array}$ & $\begin{array}{l}\text { Paget's } \\
\text { disease }\end{array}$ & $\begin{array}{l}\text { Primary } \\
\text { hyperpara- } \\
\text { thyroidism }\end{array}$ & Osteoporosis & $\begin{array}{l}\text { Hepatobiliary } \\
\text { disease }\end{array}$ \\
\hline $\mathrm{N}$ & & 24 & 31 & 12 & 66 \\
\hline Serum calcium & $2.12-2.60 \mathrm{mmol} / 1$ & $2.33(0.08)$ & $2.77(0.15)$ & $2.42(0.08)$ & $2.40 \quad(0.15)$ \\
\hline Serum phosphate & $0.78-1.40 \mathrm{mmol} / 1$ & $1.09(0.09)$ & $0.62(0.09)$ & $1.06(0.12)$ & $1.09 \quad(0.31)$ \\
\hline Parathyroid hormone $1-84$ & $<65 \mathrm{ng} / \mathrm{l}$ & $33 \quad(6)$ & $139 \quad(48)$ & $27 \quad(8)$ & $31 \quad(8)$ \\
\hline$\gamma$-Glutamyltransferase & $<33 \mathrm{U} / 1$ & $16 \quad(8)$ & 16 & $14 \quad(8)$ & $(354)$ \\
\hline Alanine aminotransferase & $<40 \mathrm{U} / 1$ & $18 \quad(4)$ & (6) & $20 \quad(7)$ & $(322)$ \\
\hline Aspartate aminotransferase & $<40 \mathrm{U} / \mathrm{l}$ & $19 \quad(4)$ & (5) & $22 \quad(5)$ & (399) \\
\hline
\end{tabular}

ferent occasions. The inter-assay coefficients of variation ranged from 3 to $3.5 \%$.

The individual values of total alkaline phosphatase and bone alkaline phosphatase are shown in figures 1 and 2 and their mean values are listed in table 1 . Both total and bone alkaline phosphatase values were obviously different in pre and postmenopausal healthy women. These data have been used in order to describe distinct normal value distributions for pre and postmenopausal women (tab. 3).

The premenopausal women were chosen as reference population for the $T$ score assessment in order to provide a precise estimate of the changes occurring after menopause and after therapy with inhibitors of bone resorption. The distributions of the $T$ values are listed in table 4. Under all conditions the $T$ score of bone alkaline phosphatase provided a better discrimination of disease groups from premenopausal controls than that of total alkaline phosphatase. Thus, $\mathrm{T}$ score values below +1 of total alkaline phosphatase were found in $30.4 \%$ of the postmenopausal women, whereas $\mathrm{T}$ scores of bone alkaline phosphatase were below +1 in only $8.7 \%$ of the subjects. A trend of rising bone alkaline phosphatase with age was found in postmenopausal women $(r=+0.15)$ and bone alkaline phosphatase was somewhat higher in women who had past menopause more than 5 years ago $(60 \pm 12$ versus $68 \pm 18 \mathrm{U} / \mathrm{l}$, $\mathrm{p}=0.10)$.

After a treatment course with a bisphosphonate in the patients with Paget's disease or primary hyperparathyroidism the percentual decreases in bone alkaline phosphatase were larger than those observed in total alkaline phosphatase (fig. 3).

An estimate of the ability to detect changes is the ratio between the mean of the changes and the SD of the changes themselves, (which is the mathematical basis of the Student $\mathrm{t}$ test) and it is defined here as precision. In all groups of patients the precision was higher for bone alkaline phosphatase (range 1.6-3.4) than for total alkaline phosphatase (range $0.5-2.4$ ).

Increases in bone markers after bisphosphonate treatment are unlikely and almost certainly related to error or inaccuracy. These increases were observed for total alkaline phosphatase but not for bone alkaline phosphatase (fig. 3).

The mean percent changes in bone alkaline phosphatase and total alkaline phosphatase in osteoporotic patients 


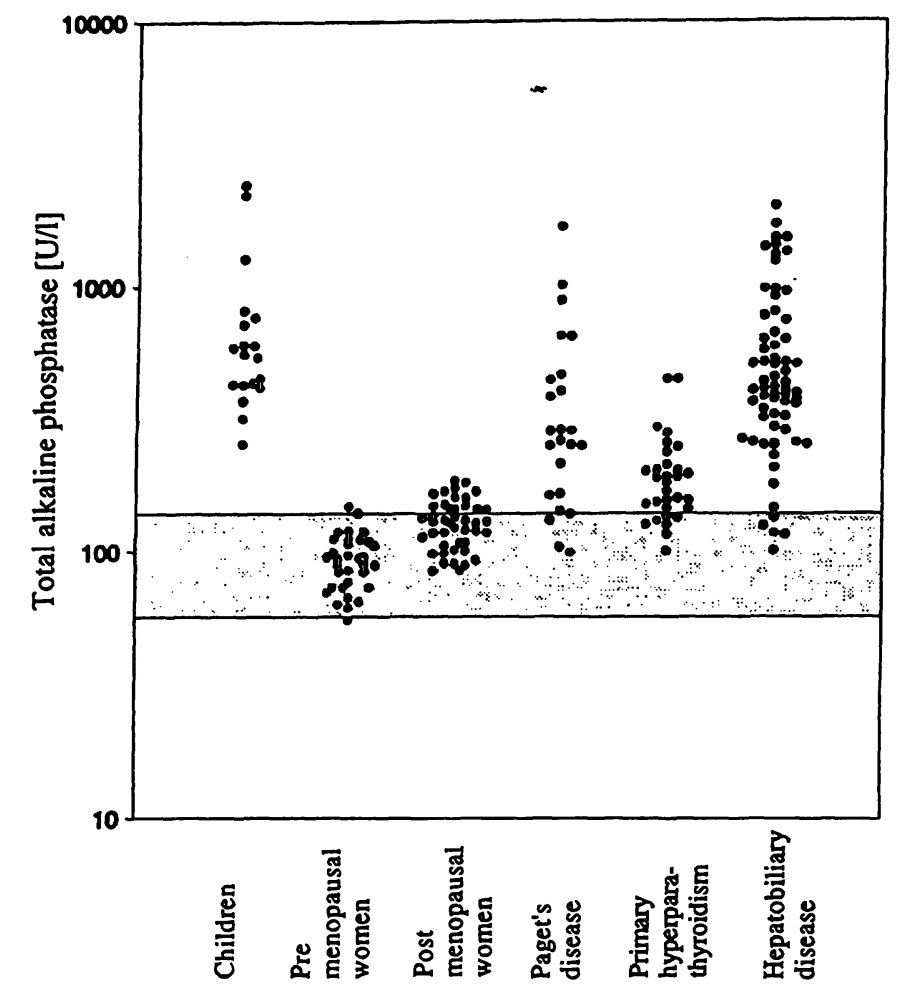

Fig. 1 Individual values (logarithmic scale) of total alkaline phosphatase in the different groups of subjects. The shadow area corresponds to the 2.5th to 97.5 th percentiles found in premenopausal women.

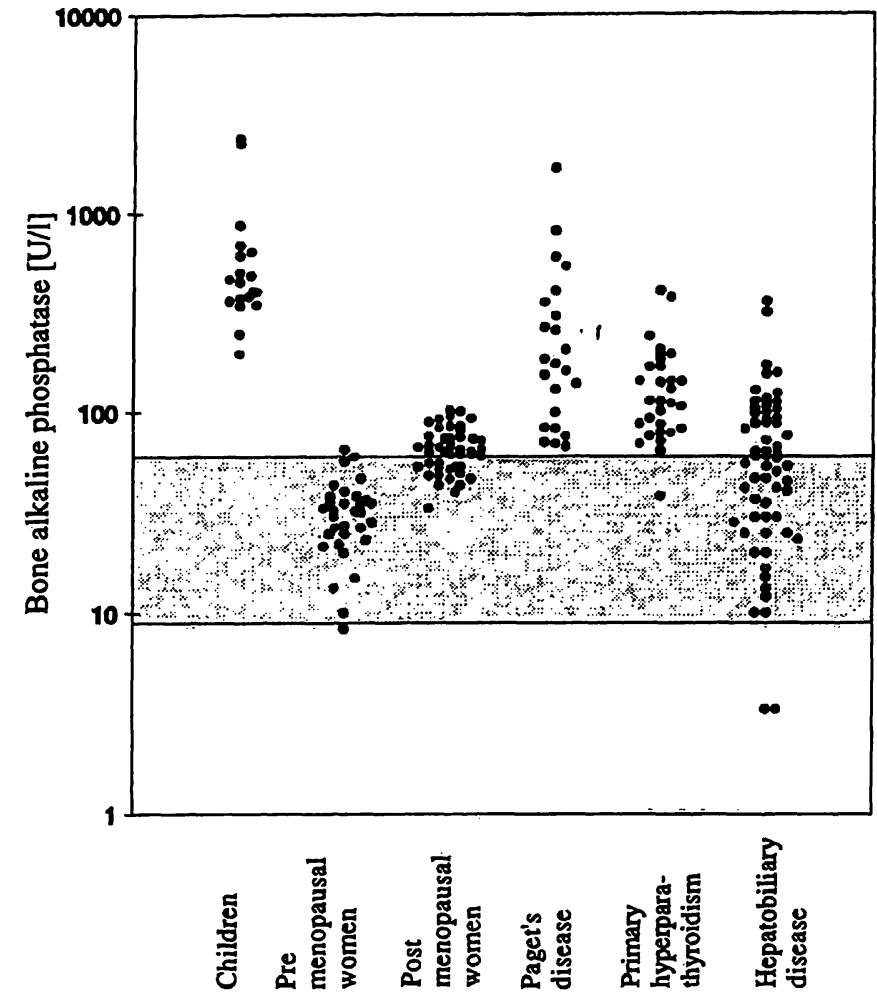

Fig. 2 Individual values (logarithmic scale) of bone alkaline phosphatase in the different groups of subjects. The shadow area corresponds to the 2.5th to 97.5th percentiles found in premenopausal women.

Tab. 3 Distribution (percentiles) of bone alkaline phosphatase and total alkaline phosphatase in apparently healthy women (all, pre- and postmenopausal)

\begin{tabular}{|c|c|c|c|c|c|c|c|c|c|c|c|c|}
\hline \multirow[t]{3}{*}{ Analyse } & \multicolumn{12}{|c|}{ Percentiles } \\
\hline & \multicolumn{4}{|c|}{ Premenopausal } & \multicolumn{4}{|c|}{ Postmenopausal } & \multicolumn{4}{|l|}{ All } \\
\hline & 50 & 90 & 95 & 97.5 & 50 & 90 & 95 & 97.5 & 50 & 90 & 95 & 97.5 \\
\hline $\begin{array}{l}\text { Bone alkaline } \\
\text { phosphatase (U/l) }\end{array}$ & 32 & 46 & 60 & 65 & 64 & 91 & 95 & 100 & 51 & 85 & 93 & 100 \\
\hline $\begin{array}{l}\text { Total alkaline } \\
\text { phosphatase (U/) }\end{array}$ & 93 & 118 & 139 & 147 & 129 & 167 & 173 & 181 & 111 & 158 & 167 & 181 \\
\hline
\end{tabular}

treated with oral alendronate are shown in figure 4. The precision ( $\eta$ mean/SD of the $\eta$ mean) was 0.7 and 1.7 for total alkaline phosphatase and bone alkaline phosphatase respectively.

Bone alkaline phosphatase was above $65 \mathrm{U} / 1$ (upper range for premenopausal women) in $42 \%$ of the cases and above $100 \mathrm{U} / \mathrm{l}$ (upper range for postmenopausal women) in $24 \%$ of the cases in patients with hepatobiliary diseases.

In patients with metabolic bone diseases the values of total alkaline phosphatase were significantly correlated with the values of alkaline phosphatase found in the supernatant corrected for dilution (supernatant alkaline phosphatase $=55+0.15$ total alkaline phosphatase; $\mathrm{r}=0.65 ; \mathrm{p}<0.001)$ and in patients with hepatobiliary diseases in significant correlation was found between total alkaline phosphatase and the difference between total and supernatant enzyme activity (precipitated alkaline phosphatase $=29+0.08$ total alkaline phosphatase; $r=0.53 ; p<0.001$ ). Thus, approximately $15 \%$ of alkaline phosphatase might not be precipitated by lectin in patients with bone disease and $8 \%$ might be aspecifically precipitated in hepatobiliary patients. Interestingly the intercepts of the functions are similar to the normal premenopausal values for bone and residual alkaline phosphatase. When bone alkaline phosphatase was calculated by adopting the function suggested in the commercial kit, total alkaline phosphatase and bone alkaline phosphatase were insignificantly related (bone alkaline 


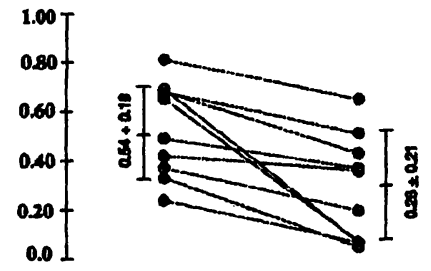

Pagete disease
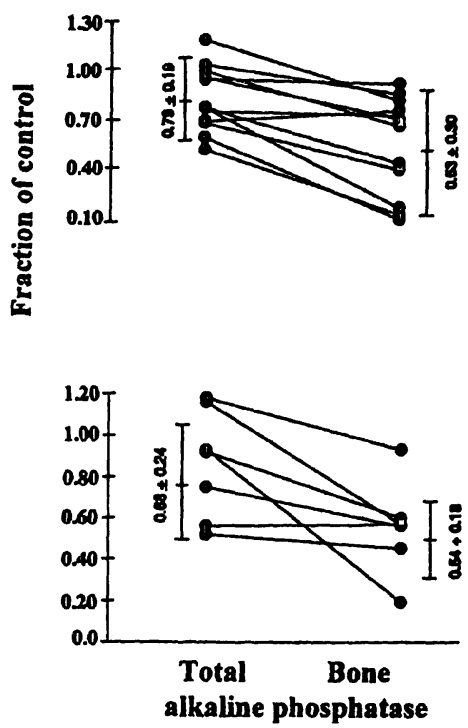

Primary hyperparathyroidism (long-term effect)

Fig. 3 Individual and mean ( $\pm S D$ ) fractional changes in bone alkaline phosphatase and total alkaline phosphatase in patients with metabolic bone diseases treated with intravenous alendronate. The patients with primary hyperparathyroidism were studied either within 3 weeks (middle panel) or within 2 months (lower panel).

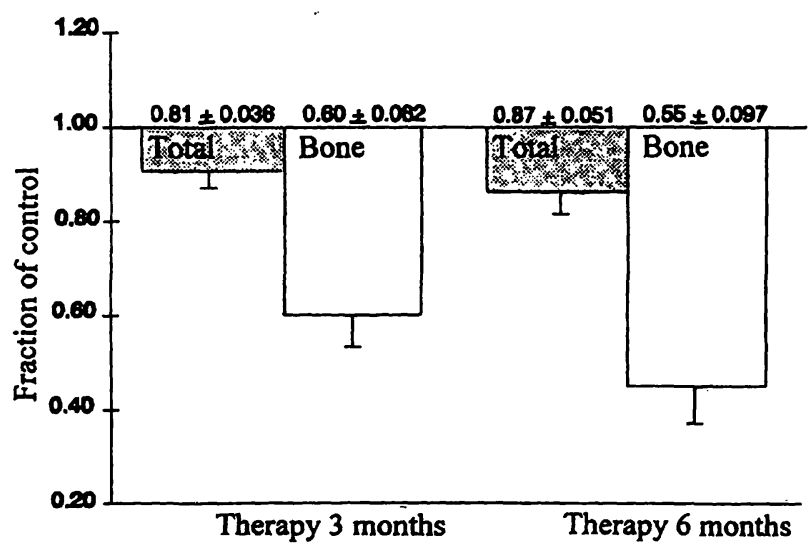

Fig. 4 Mean fractional changes ( \pm SE) in bone and total alkaline phosphatase after 3 and 6 months of therapy with oral alendronate of osteoporotic patients.

phosphatase $=0.03$ total alkaline phosphatase +35 , $r=0.26 ; p<0.1$ ) in patients with hepatobiliary disease without apparent bone involvement. Residual alkaline phosphatase was also insignificantly related to total alkaline phosphatase (residual alkaline phosphatase $=0.03$ total alkaline phosphatase $+59 ; \mathrm{r}=0.27$; $\mathrm{p}<0.1)$ in patients with metabolic bone diseases. The latter correlation is principally driven by the samples with total alkaline phosphatase well above 500-1000

$\mathrm{U} / \mathrm{l}$ and this might indicate that in patients with the highest values of alkaline phosphatase the bone fraction of alkaline phosphatase is not fully precipitated by lectin.

Three serum samples from patients with severe Paget's disease without apparent hepatobiliary failure were sequentially diluted with either serum with undetectable alkaline phosphatase activity (heath inactivated normal serum) or saline solution from $1: 1.5$ to $1: 32$ before measurements. An example of the results obtained is shown in figure 5. It appears that in undiluted serum samples with bone alkaline phosphatase values above $1000 \mathrm{U} / \mathrm{l}$ the bone fraction is underestimated. Dilution down to levels of bone alkaline phosphatase below $\sim 1000 \mathrm{U} / \mathrm{l}$ restores linearity and residual alkaline phosphatase is that expected from patients without hepatobiliary diseases (fig. 5).

A strict correlation $(r=0.88)$ was found between the bone alkaline phosphatase values measured in 32 patients by both wheat-germ lectin preiciptation and IRMA (data not shown).
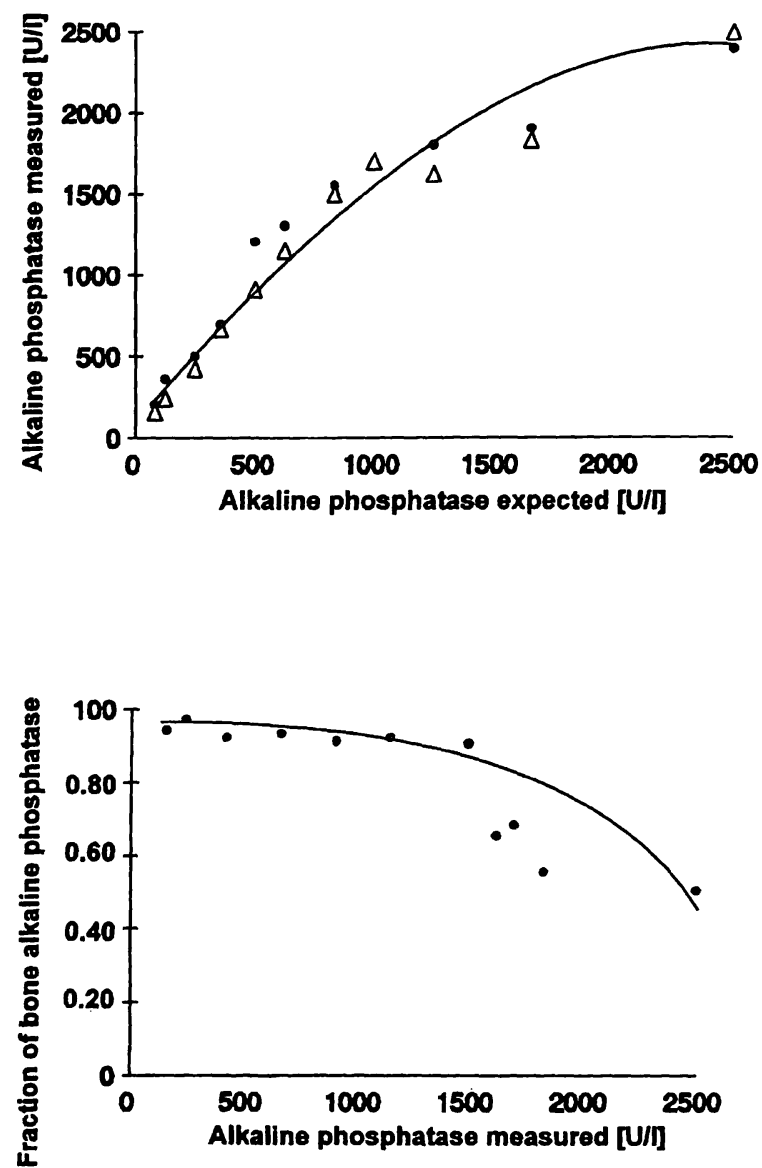

Fig. 5 Correlation (upper panel) of the measured and calculated bone alkaline phosphatase activity from a serum sample of a patient with severe Paget's disease diluted either with saline solution (open triangles) or heat-inactivated serum (solid dots).

The proportion of bone alkaline phosphatase over total alkaline phosphatase falls below $85-95 \%$ for bone alkaline phosphatase values above $1000 \mathrm{U} / \mathrm{l}$ (lower panel). 


\section{Discussion}

Serum alkaline phosphatase is extensively used for assessing bone turnover in patients with metabolic bone diseases, but its utility is often impaired by changes occurring in non-bone alkaline phosphatase. Here we used the lectin precipitation technique to measure the bone isoforms in normal subjects and in patients with metabolic bone diseases as well as liver diseases.

Heat inactivation has been routinely employed for some time, but the method is both inaccurate and imprecise and one might wonder whether gains in term of accuracy are lost in terms of precision (4). If this is the case, the utility of this method should be limited to patients suspected to have abnormal non-bone serum alkaline phosphatase. With the lectin precipitation technique applied in this investigation we have shown that the discrimination of subjects with high bone turnover, including children, patients with primary hyperparathyroidism or Paget's disease from normals were more accurate with bone alkaline phosphatase evaluation if compared to total alkaline phosphatase.

In postmenopausal women the $T$ score (tab. 4) was within $90 \%$ of confidence limit of healthy young subjects in $22 \%$ of the cases for total alkaline phosphatase and in $6 \%$ for bone alkaline phosphatase $(\mathrm{p}<0.001)$. The difference between pre- and postmenopausal women for bone alkaline phosphatase reflects the wellknown phenomenon of increased postmenopausal bone turnover and it is so striking that one may wonder whether two normative values should (tab. 3) be adopted for pre- and postmenopausal women. It has been recently reported (11) that vertebral bone density is significantly correlated with lectin-precipitated bone alkaline phosphatase, but not with total alkaline phosphatase. This suggests that bone alkaline phosphatase may also provide useful information regarding the inter-individual variance in bone turnover and bone loss in postmenopausal women.

Bone alkaline phosphatase also seems to provide advantages over total alkaline phosphatase in monitoring the effect of inhibitors of bone resorption given to patients with either Paget's disease or primary hyperparathyroidism. The changes in bone alkaline phosphatase were both greater and more precise. Since total and unprecipitated enzyme activity was measured by the same colorimetric method, these results indicate that the improved precision has to be attributed to fluctuation over time of non-bone alkaline phosphatase activity, which represents a source of error when total alkaline phosphatase is estimated. The changes occurring in patients with postmenopausal osteoporosis treated chronically with a bisphosphonate can also be better monitored by measuring bone alkaline phosphatase as compared to total alkaline phosphatase (fig. 4). Thus, the ratio between the mean changes and its standard deviation is approximately 2.5 for total alkaline phosphatase and 6 for bone alkaline phosphatase. This is of particular importance in clinical therapeutic trials of osteoporosis, where the absolute expected changes are usually small.

In patients with bone diseases a correlation was found between total enzyme activity and residual alkaline phosphatase, which was mainly due to cases with enzyme activity above $1000 \mathrm{U} / \mathrm{l}$. The results of the linearity tests we carried-out are somewhat at variance with those reported by others $(6,7,12)$. Behr \& Barnert showed good linearity for a serum sample containing only $400 \mathrm{U} / 1$ of bone alkaline phosphatase but, in agreement with our results, they claim (the data are not shown) that for bone alkaline phosphatase activities $>1200 \mathrm{U} / 1$ the lectin concentration had to be proportionally increased for complete precipitation of bone alkaline phosphatase. It has also been recommended not to dilute the serum samples with saline

Tab. 4 T score (mean and distribution in postmenosausal women, in children, in patients with Paget's disease and with primary hyperparathyroidism

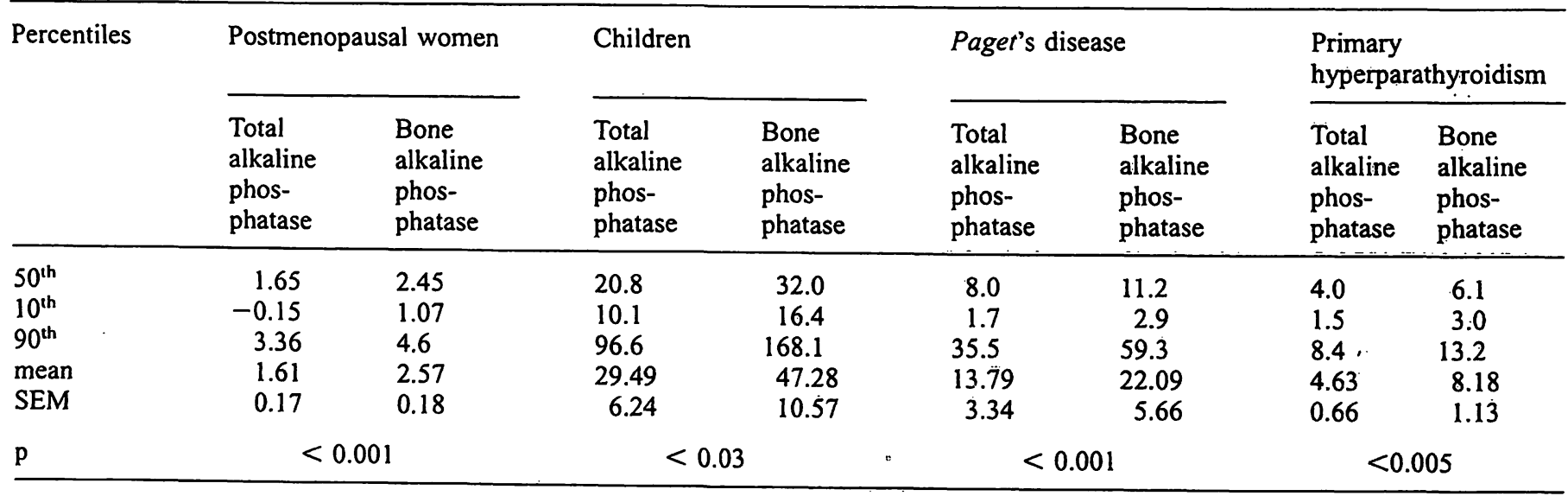


solution (6) but we could not find linearity differences when samples were diluted with saline solution or serum (fig. 5). In previous studies the highest bone alkaline phosphatase tested samples contained $<1000$ $\mathrm{U} / \mathrm{l}$. Our results indicate that for such concentrations linearity is preserved, but in patients with severe metabolic bone diseases the precipitation of bone alkaline phosphatase by lectin is not complete and sample dilution is mandatory if bone alkaline phosphatase is expected to be higher than $1000 \mathrm{U} / \mathrm{l}$ (fig. 5).

From the function relating total alkaline phosphatase to supernatant and precipitated alkaline phosphatase in patients with bone and hepatobiliary diseases respectively it can be estimated that approximately $15 \%$ of the bone isoenzyme is not precipitated and $8 \%$ of non-bone alkaline phosphatase is unspecifically precipitated by lectin. These results are similar to those found by comparative studies between lectin precipitation and electrophoretic method ( $10 \%$ and $5 \%$ respectively) and with the correction function (7) suggested in the commercial kit was

\section{References}

1. Farley JR, Baylink DJ. Skeletal alkaline phosphatase activity as a bone formation index in vitro. Metabolism 1986; 35:563-71.

2. Delmas PD. Biochemical markers of bone turnover for the clinical assessment of metabolic bone disease. Endocrinol Metab Clin North Am 1990; 19:1-18.

3. Weiss MJ, Henthorn PS, Lafferty MA, Slaughter C, Raducha $M$, Harris $H$. Isolation and characterisation of a cDNA encoding a human liver/bone/kidney-type alkaline phosphatase. Proc Natl Acad Sci USA 1986; 83:7182-6.

4. Price CP. Multiple forms of human serum alkaline phosphatase: detection and quantitation. Ann Clin Biochem 1993; 30:355-72.

5. Moss DW. Henderson enzymes. In: Burts CA, Ashwood ER, editors. Tietz textbook of clinical chemistry. Philadelphia: Saunders, 1994:735-896.

6. Rosalki SB, Foo AY. Two new methods for separating and quantifying bone and liver alkaline phosphatase isoenzymes in plasma. Clin Chem 1984; 30:1182-7.

7. Rosalki SB, Foo AY, Burlina A, Prellwitz W, Stieber P, Neumeier D, et al. Multicenter evaluation of iso-ALP test kit for measurement of bone alkaline phosphatase activity in serum and plasma. Clin Chem 1993; 39/4:648-52.

8. "Optimierte Standardmethode" der Deutschen Gesellschaft für Klinische Chemie. J Clin Chem Clin Biochem 1972; 10:182-92.

9. Marshall RW. Plasma fractions. In: Nordin BEC, editor. Calcium phosphate and magnesium metabolism. Edinburgh Churchill Livingstone. 1976: 162-85. based. When this correction is applied, bone alkaline phosphatase results may still be unexpectedly increased in hepatobiliary patients (1. c. (12) and our results). From the correlation found between total and bone alkaline phosphatase this overestimate might be approximately $3 \%$. With a recently developed immunological method $(12,14)$ the degree of cross-reactivity between bone and non-bone alkaline phosphatase has been found to be $16 \%$, considerably higher than that we found by lectin precipitation. This might be a problem in patients with hepatic diseases, arousing the erroneous suspicion of abnormal bone involvement.

In conclusion the results of the present study indicate that the measurement of bone alkaline phosphatase by wheat germ agglutinin separation, a method also easily available to any small laboratory, can be considered a substantial improvement over total alkaline phosphatase measurement with respect to accuracy and is useful for monitoring treatment-related changes in patients with metabolic bone diseases.

10. Rossini M, Gatti D, Zamberlan N, Braga V, Dorizzi R, Adami $S$. Long-term effects of a treatment course with oral alendronate of postmenopausal osteoporosis. J Bone Miner Res 1994; 9:1833-7.

11. Johnston JD, Koneru S, Kuwana T, Rosalki SB. Bone-derived serum enzymes and bone density in perimenopausal Caucasian women. Ann Clin Biochem 1993; 30:191-4.

12. Behr W, Barnert J. Quantification of bone alkaline phosphatase in serum by precipitation with wheat-germ lectin: a simplified method and its clinical plausibility. Clin Chem 1986; 32:1960-6.

13. Garnero P, Delmas PD. Assessment of the serum levels of bone alkaline phosphatase with a new immunoradiometric assay in patients with metabolic bone disease. J Clin Endocrinol Metab 1993; 77:1046-53.

14. Garnero P, Shih WJ, Gineyts E, Karpf DB, Delmas PD. Comparison of new biochemical markers of bone turnover in late postmenopausal osteoporotic women in response to alendronate treatment. J Clin Endocrinol Metab 1994; $79: 1693-1700$

Prof. Silvano Adami

Cattedra di Reumatologia

Policlinico

$1-37134$ Verona

Italy 
